\title{
INDIVIDUAL MONITORING AT ACCELERATOR CENTRES
}

Christian Wernli*

Paul Scherrer Institute, 5232 Villigen, Switzerland

\begin{abstract}
The accurate determination of personal dose equivalent requires the proper use of appropriate radiological quantities and units, knowledge of the dose equivalent response of the personal dosemeters used and detailed information on the fluence as well as dose equivalent spectra at the workplaces. This information can then be used to select the appropriate dosemeters, to set up the optimum calibration conditions and to introduce, in case of need, normalisation factors for application in specific radiation fields. High-energy neutrons contribute significantly to the radiation fields around high-energy particle accelerators. Examples for procedures and methods to determine personal dose equivalent at accelerator centres are given.
\end{abstract}

\section{INTRODUCTION}

The development of dosimetric concepts and the definition of specific quantities for use in radiation protection have a long history. The International Commission on Radiation Units and Measurements (ICRU) and the International Commission on Radiological Protection (ICRP) are the key organisations in this field. In 2007, in its Publication $103^{(1)}$, the ICRP has published its most recent general recommendations for radiation protection. These recommendations include basic concepts and guidelines for radiation protection, a concept for protection quantities and operational quantities, as well as limits for exposure. In addition, internationally agreed conversion coefficients are necessary for implementation of the operational quantities into practice. In the following, these concepts and methods are described for applications around accelerators.

The primary beam of an accelerator consists in most cases of protons or electrons, rarely of heavier ions. In general, the number of primary particles penetrating the shielding is, even at very high energies, negligible. The resulting types of secondary radiation in occupied spaces around an accelerator are normally photons, neutrons, electrons and muons. Most of these radiations may result from interactions of the primary beam, but some may be the result of induced radioactivity. For individual monitoring around accelerators, photon and neutron radiations are of main importance ${ }^{(2,3)}$.

Various methods and techniques are used for individual monitoring. Around high-energy particle accelerators, dedicated dosimetry systems need to be applied, especially for high-energy neutron radiation. Besides established systems, the development of new techniques is in progress and may lead to new solutions.

*Corresponding author: christian.wernli@psi.ch

\section{CONCEPT OF RADIATION PROTECTION QUANTITIES}

The international commissions ICRP and ICRU have developed a hierarchy of quantities for radiation protection applications which can be described by protection quantities taking account of human body properties and operational quantities for monitoring of external exposure.

The basic idea of a protection quantity is to relate the "risk" of exposure to ionising radiation (exposure by internal and external radiation sources) to a single (dose) quantity which takes account of the man as a receptor, the different radiation sensitivities of various organs and tissues and the different radiation qualities. Other influence parameters, however, e.g. the influence of dose and dose rate or sex and age of a person exposed on the biological response to the exposure risk, were not considered in the definition of these quantities. These effects are to some extent taken into account by the various limitations of exposure.

Operational quantities are dose quantities defined for use in radiation protection measurements for external exposure (area or individual monitoring). They usually should provide an estimate of or an upper limit for the value of the limiting protection quantities due to an exposed, or potentially exposed, person and are often used in place of those quantities in practical regulations. Operational quantities are needed for monitoring external exposures because protection quantities generally are not measurable.

Both, protection quantities and operational quantities can be related to "basic physical quantities" as specified in ICRU Reports 33, 39, 43 and $51^{(4-7)}$, which are generally used in radiation metrology and in radiation dosimetry in particular and are defined without considering any specific aspect of radiation protection. Basic physical quantities are quantities whose units are directly obtained through primary standards at national standards laboratories, e.g. air 


\section{INDIVIDUAL MONITORING AT ACCELERATOR CENTRES}

kerma for photons, fluence for neutrons and absorbed dose for beta radiation.

Most of the radiation protection quantities mentioned above either are not directly measurable or their values are not directly traceable to primary standards. For this reason, knowledge of the numerical relations between the basic physical quantities and these dose quantities is a very important condition for the practical implementation of the whole system of radiation protection quantities.

Therefore, it is vital that an agreed set of data accepted by national and international authorities is available. Following ICRP Publication $60^{(8)}$, conversion coefficients for photon, neutron and electron radiation were evaluated and published in ICRP Publication $74^{(9)}$ in 1996 . So far, no newly calculated conversion coefficients based on ICRP Publication 103 have been published by ICRU or ICRP. Calculations by Schlattl et al. ${ }^{(10)}$ have shown that the effect for photons are expected to be modest. It is recommended to continue to use the coefficients of ICRP Publication 74 for the time being. For neutron radiation, a significant change in effective dose conversion coefficients $\left(E / H_{\mathrm{p}}(10), E / H^{*}(10)\right)$ is expected. New calculations are needed to provide consistent results.

\section{PROTECTION QUANTITIES}

\section{Absorbed dose and equivalent dose in an organ or tissue}

The absorbed dose in an organ or tissue, $D_{\mathrm{T}}$, is the absorbed dose averaged over the volume of a tissue or organ $\mathrm{T}$ (rather than at a point). While the absorbed dose at a point generally is the fundamental dose quantity, in radiation protection, the mean dose in an organ becomes the basic protection quantity correlated with the exposure risk. This concept, therefore, is obviously based on the linear doseeffect relationship and the additivity of doses for risk assessment as an appropriate approximation in the low-dose region. Otherwise, averaging of doses and adding of doses over long periods would not be an acceptable procedure.

The equivalent dose in an organ or tissue is defined by

$$
H_{\mathrm{T}}=\Sigma w_{\mathrm{R}} D_{\mathrm{T}, \mathrm{R}}
$$

where $D_{\mathrm{T}, \mathrm{R}}$ is the mean organ dose in the organ or tissue $T$ from radiation of type $R$ incident on the human body and $w_{\mathrm{R}}$ are radiation weighting factors characterising the biological effectiveness of the specific radiation $\mathrm{R}$ relative to photons. The sum is taken over all types of radiation involved.

\section{Radiation weighting factors}

For external irradiation, the values of the radiation weighting factors are given by the parameters of the external radiation field only (by the type and spectral distribution of the radiation incident on the body). This means that $w_{\mathrm{R}}$ is a body-averaged value representing a mean value for the relative biological effectiveness of all tissues of the body. Because the $w_{\mathrm{R}}$ value is the same for all organs in a body, this procedure obviously ignores any local variation of the radiation quality in the human body that may result from the generation of secondary radiation of different types in the human body. This effect is important mainly in the case of incident neutrons where secondary photons strongly contribute to the absorbed doses of various organs.

The $w_{\mathrm{R}}$ values for various types of radiation are specified in ICRP Publication 103. For photons, electrons and muons of all energies, a value of 1 is fixed with the exception of Auger electrons emitted from nuclei bound to DNA, but this is of no importance for external irradiation. For protons and charged pions, the value of 2 is recommended. This replaces the $w_{\mathrm{R}}$ for protons of 5 given in ICRP Publication 60. The radiation weighting factor for neutrons depends on the neutron energy. A continuous $w_{\mathrm{R}}$ function has been introduced in ICRP Publication 103:

$$
w_{\mathrm{R}}=\left\{\begin{array}{lc}
2.5+18.2 \exp \left(-\frac{1}{6}\left[\ln \left(E_{\mathrm{n}}\right)\right]^{2}\right), & E_{\mathrm{n}}<1 \mathrm{MeV} \\
5.0+17.0 \exp \left(-\frac{1}{6}\left[\ln \left(2 E_{\mathrm{n}}\right)\right]^{2}\right), & 1 \mathrm{MeV} \leq E_{\mathrm{n}} \leq 50 \mathrm{MeV} \\
2.5+3.25 \exp \left(-\frac{1}{6}\left[\ln \left(0.04 E_{\mathrm{n}}\right)\right]^{2}\right), & E_{\mathrm{n}}>50 \mathrm{MeV}
\end{array}\right.
$$

In ICRP Publication 60, different $w_{\mathrm{R}}$ values were given by a step function. For comparison, both sets of data (ICRP 60 and ICRP 103) are given in Table 1 and Figure 1.

The conversion coefficients for neutrons published in ICRP Publication 74 are based on an approximate continuous function published in ICRP Publication 60. This approximate function is not to be confused with the new function of ICRP Publication 103!

\section{Effective dose}

The effective dose, $E$, is the weighted sum of organ equivalent doses:

$$
E=\Sigma w_{\mathrm{T}} H_{\mathrm{T}} \quad \text { with } \Sigma w_{\mathrm{T}}=1,
$$

where $w_{\mathrm{T}}$ are tissue weighting factors characterising the relative sensitivity of the various tissues with respect to cancer induction and mortality. In ICRP Publication 103, 15 tissues and organs are specified with individual weights $w_{\mathrm{T}}$ (see Table 2). 
Table 1. Radiation weighting factors, $w_{R}$, for different types of radiation.

\begin{tabular}{lll}
\hline \multirow{2}{*}{ Radiation } & \multicolumn{2}{c}{ Radiation weighting factor $w_{\mathrm{R}}$} \\
\cline { 2 - 3 } & ICRP 60 & ICRP 103 \\
\hline Electrons $^{\mathrm{a}}$, muons & 1 & 1 \\
Photons & 1 & 1 \\
Neutrons & 5 & Recommended $w_{R}$ function shown in Figure 1 \\
$E_{\mathrm{n}}<10 \mathrm{keV}$ & 10 & \\
$E_{\mathrm{n}}=10 \mathrm{keV}$ to $100 \mathrm{keV}$ & 20 & \\
$E_{\mathrm{n}}>100 \mathrm{keV}$ to $2 \mathrm{MeV}$ & 10 & \\
$E_{\mathrm{n}}>2 \mathrm{MeV}$ to $20 \mathrm{MeV}$ & 5 & 2 \\
$E_{\mathrm{n}}>20 \mathrm{MeV}$ & 5 & 20 \\
Protons & 20 & \\
$\alpha$-Particles, fission fragments, heavy ions & &
\end{tabular}

${ }^{\mathrm{a}}$ With the exception of Auger electrons from atoms bound to DNA.

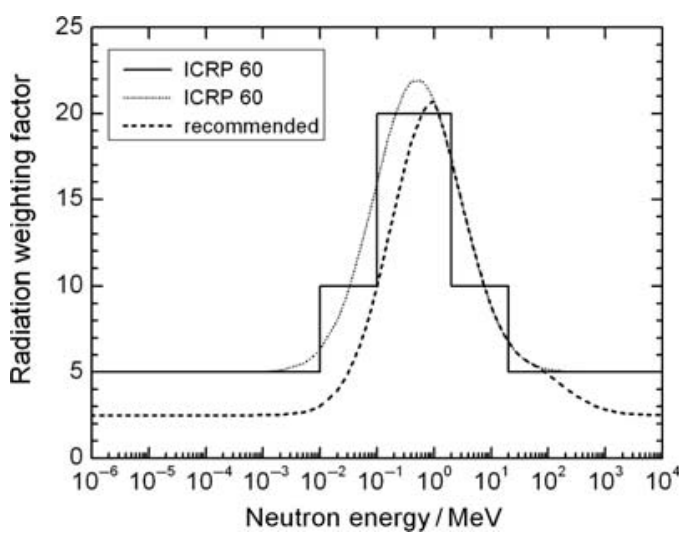

Figure 1. Radiation weighting factor, $\mathrm{w}_{\mathrm{R}}$, for neutrons versus neutron energy. Continuous function recommended in ICRP Publication 103 and step function and continuous function given in Publication 60.

Table 2. ICRP recommendations for tissue weighting factors, $w_{\mathrm{T}}$, in Publication 103.

\begin{tabular}{lcc}
\hline Organ/tissue & $w_{\mathrm{T}}$ & $\Sigma w_{\mathrm{T}}$ \\
\hline $\begin{array}{l}\text { Lung, stomach, colon, bone } \\
\text { marrow, breast, remainder }\end{array}$ & 0.12 & 0.72 \\
$\begin{array}{l}\text { Gonads } \\
\text { Thyroid, oesophagus, bladder, liver }\end{array}$ & 0.08 & 0.08 \\
$\begin{array}{l}\text { Bone surface, skin, brain, salivary } \\
\text { glands }\end{array}$ & 0.04 & 0.16 \\
Total & & 0.04 \\
\hline
\end{tabular}

The number of selected tissues and organs and some weighting factors are different in ICRP Publication 60. For details, see ICRP Publication 103.

\section{OPERATIONAL QUANTITIES}

The concept of operational quantities

The basic concept of the operational quantities is described in the ICRU Reports 39 and 43. The present definitions are given in ICRU Report 51. The radiation incident on a human body is characterised as penetrating radiation or low-penetrating radiation, depending on the ratio of the skin dose to effective dose. Radiation is considered to be low-penetrating when the dose equivalent received by the skin (dose received at a depth of $0.07 \mathrm{~mm}$ ) in the case of normal incidence of a broad radiation beam is higher than ten times the effective dose; otherwise, it is considered to be penetrating. Low-penetrating radiations are $\alpha$ particles, $\beta$-particles with energies below $2 \mathrm{MeV}$ and photons with energies below about $12 \mathrm{keV}$. Neutrons always are penetrating radiation.

Due to the different tasks in radiation protection monitoring - area monitoring for controlling the radiation at workplaces and definition of controlled areas or individual monitoring for the control and limitation of individual exposures - different operational quantities were defined. While measurements with an area monitor are mostly performed free in air, an individual dosemeter is usually worn on the front of the body. As a consequence, in a given situation, the radiation field "seen" by an area monitor free in air differs from that "seen" by an individual dosemeter worn on a body where the radiation field is strongly influenced by the backscatter and absorption of radiation in the body. The operational quantities allow for this effect.

\section{Dose equivalent and quality factor}

The quantity dose equivalent, $H$, has been defined by

$$
H=Q D,
$$




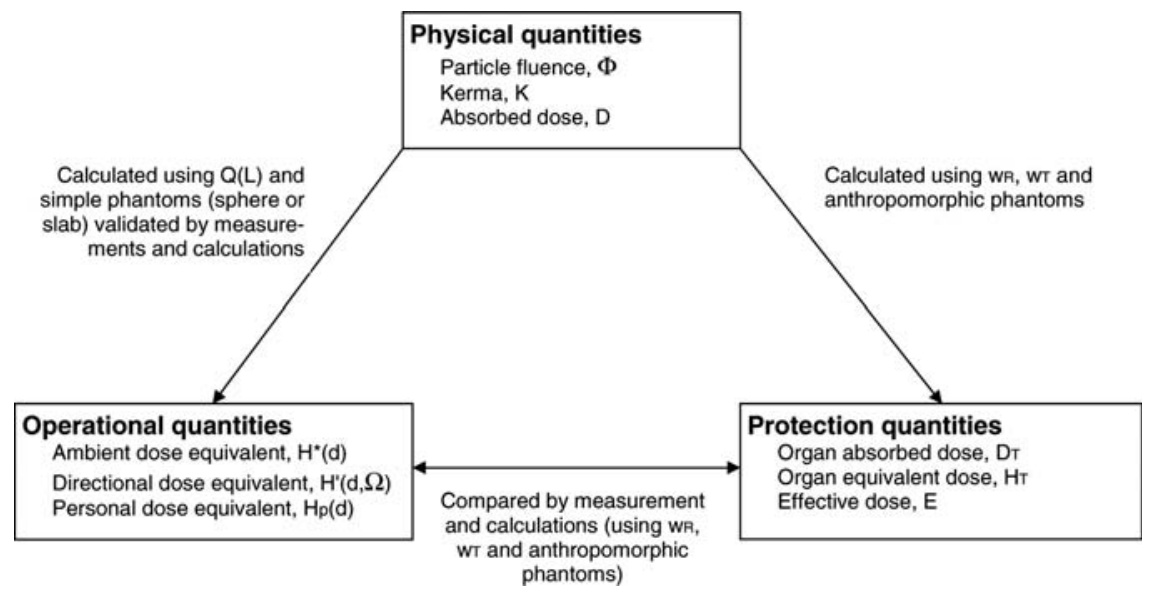

Figure 2. Relationship between the basic physical quantities, the operational quantities and the protection quantities ${ }^{(8)}$.

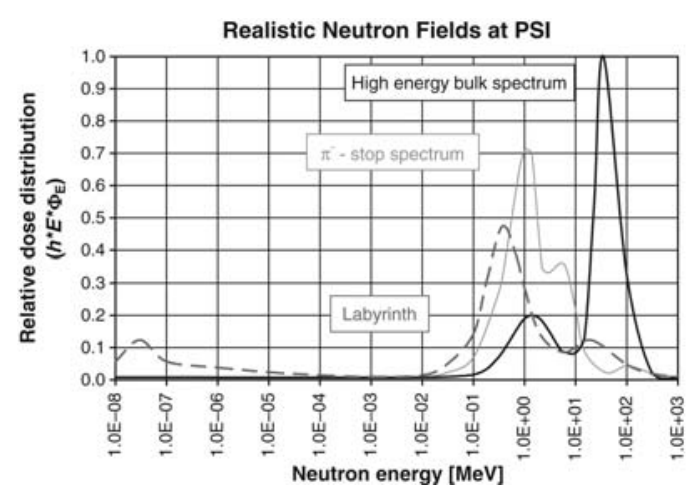

Figure 3. Relative neutron dose distribution versus energy in different areas of the experimental hall at the PSI $590 \mathrm{MeV}$ proton accelerator.

where $D$ is the absorbed dose at the point of interest and $Q$ a quality factor weighting the relative biological effectiveness of radiation. $Q$ is defined as a function of the linear energy transfer, $L$, of a charged particle in water. Dose equivalent is restricted to the definition of operational radiation protection quantities and the quality factor function $Q(L)$ is given by the following equation:

$Q(L)=\left\{\begin{array}{cc}1 & \text { for } L<10 \mathrm{keV} \mathrm{\mu m}^{-1} \\ 0.32 L-2.2 & \text { for } 10 \leq L \leq 100 \mathrm{keV} \mu \mathrm{m}^{-1} \\ \frac{300}{\sqrt{L}} & \text { for } L>100 \mathrm{keV} \mathrm{\mu m}^{-1}\end{array}\right.$

The quality factor $Q$ at a point in tissue is then given by ref. ${ }^{(7)}$

$$
Q=\frac{1}{D} \int_{0}^{\infty} Q(L) D_{L} \mathrm{~d} L
$$

where $D_{\mathrm{L}}$ is the distribution of $D$ in $L$ at the point of interest. This function is most important for neutrons because various types of secondary charged particles are produced in tissue in this case.

\section{Operational quantities for area monitoring}

\section{The ICRU sphere phantom}

For all types of radiation, the operational quantities for area monitoring are defined on the basis of a phantom, the ICRU sphere. It is a sphere of tissueequivalent material (diameter: $30 \mathrm{~cm}$, density: $1 \mathrm{~g} \mathrm{~cm}^{-3}$, mass composition: $76.2 \%$ oxygen, $11.1 \%$ carbon, $10.1 \%$ hydrogen and $2.6 \%$ nitrogen). It adequately approximates the human body as regards the scattering and attenuation of the radiation fields under consideration.

The operational quantities for area monitoring defined in the ICRU sphere should retain their character of a point quantity and the property of additivity. This is achieved by introducing the terms expanded and aligned radiation field in the definition of these quantities (see ICRU Report 39) ${ }^{(5)}$.

\section{Ambient dose equivalent, $H^{*}(d)$}

For area monitoring of penetrating radiation, the operational quantity is the ambient dose equivalent, $H^{*}(d)$, with $d=10 \mathrm{~mm}$. The ambient dose equivalent, $H^{*}(d)$, at a point of interest in the real radiation field, is the dose equivalent that would be produced 


\section{WERNLI}

by the corresponding aligned and expanded radiation field, in the ICRU sphere at a depth $d$, on the radius vector opposing the direction of radiation incidence. For penetrating radiation, it is $d=10 \mathrm{~mm}$ and $H^{*}(d)$ is written as $H^{*}(10) . H^{*}(10)$ should give a conservative estimate of the effective dose a person would receive when staying at this point.

For data given in ICRP Publications 60 and 74, this is always the case for photons below $10 \mathrm{MeV}$. For neutrons, the situation is different. $H^{*}(10)$ is not conservative with respect to $E$ under anterior/ posterior (AP) irradiation conditions in the energy range from $1 \mathrm{eV}$ to about $50 \mathrm{keV}$. In realistic neutron fields with a broad neutron energy distribution, however, this energy range is often of limited importance and in practice $H^{*}(10)$, therefore, remains in most cases conservative with respect to $E$. For further details, see refs ${ }^{(11-13)}$.

\section{Directional dose equivalent, $H^{\prime}(d, \Omega)$}

For area monitoring of low-penetrating radiation, the operational quantity is the directional dose equivalent, $H^{\prime}(d, \Omega)$ with $d=0.07 \mathrm{~mm}$ or, in rare cases, $d=3 \mathrm{~mm}$. The directional dose equivalent, $H^{\prime}(d, \Omega)$, at a point of interest in the actual radiation field, is the dose equivalent that would be produced by the corresponding expanded radiation field, in the ICRU sphere at a depth $d$, on a radius in a specified direction $\Omega$.

For low-penetrating radiation, $d$ is equal to $0.07 \mathrm{~mm}$ and $H^{\prime}(d, \Omega)$ is written as $H^{\prime}(0.07, \Omega)$.

\section{Operational quantities for individual monitoring, $H_{\mathrm{p}}(d)$}

Individual monitoring is usually performed with dosemeters worn on the body and the operational quantity defined for this application takes this situation into account. For individual monitoring, the operational quantity is the personal dose equivalent, $H_{\mathrm{p}}(d)$. The personal dose equivalent, $H_{\mathrm{p}}(d)$, is the dose equivalent in ICRU tissue at a depth $d$ in a human body below the position where an individual dosemeter is worn. For monitoring of the skin dose $d=0.07 \mathrm{~mm}$ is recommended and for monitoring effective dose $d=10 \mathrm{~mm}$. The operational quantities for individual monitoring meet several criteria. They are equally defined for all types of radiation, additive with respect to various directions of radiation incidence, take into account the backscattering from the body and can be approximately measured with a dosemeter worn on the body. The personal dose equivalent quantities, $H_{\mathrm{p}}(10)$ and $H_{\mathrm{p}}(0.07)$, are defined in the person, in the actually existing radiation field, and are measured directly on the person.

Other requirements the quantities should satisfy can, however, be fulfilled only with additional specifications. Obviously, the person influences the radiation field by scattering and attenuating the radiation. Since $H_{\mathrm{p}}(10)$ and $H_{\mathrm{p}}(0.07)$ are defined in the body of each person considered, their values vary from one person to another and also depend on the location on the body where the dosemeter is worn. In a non-isotropic radiation field, the value of personal dose equivalent also depends on the orientation of the person in this field.

An operational quantity for individual monitoring should allow the effective dose to be assessed or should provide a conservative estimate under nearly all irradiation conditions. This obviously is not always possible. For example, if a dosemeter is worn at the front side of the body and the person is exposed from the back, this condition cannot be fulfilled because most of the radiation will already be absorbed within the body and not reach the front where the dosemeter is positioned. Even if the dosemeter correctly measures $H_{\mathrm{p}}(10)$ in this case, this value is not a conservative estimate of the effective dose, $E$. It is, therefore, an additional requirement in individual dosimetry that the personal dosemeter must be worn at a position on the body which is representative of body exposure. For a dosemeter position in front of the trunk, however, the quantity $H_{\mathrm{p}}(10)$ mostly furnishes a conservative estimate of $E$ even in cases of lateral or isotropic radiation incidence on the body. A further requirement for an operational quantity is that it allows dosemeters to be calibrated under reference conditions in terms of that quantity. The personal dose equivalent is defined in the individual human body and it is obvious that individual dosemeters cannot be calibrated in front of a real human body. For a calibration procedure, the human body must therefore be replaced by an appropriate phantom.

The conversion coefficients relating the numerical values of these quantities are published in ICRP Publication 74 based on data in ICRP Publication 60 . Recent data are also given in refs ${ }^{(10-13)}$. The relationship between the quantities is shown in Figure 2.

\section{PROCEDURES FOR CALIBRATION}

\section{Calibration of personal dosemeters ${ }^{(14)}$}

The calibration of personal dosemeters is performed with the dosemeters mounted on an appropriate phantom. Three phantoms have been defined by $\mathrm{ISO}^{(15-18)}$ for calibrations, corresponding to the positions on which personal dosemeters are worn (on the body, on the arm or on a finger). Their shapes are the same as those of the ICRU-tissue phantoms used for the calculation of the conversion coefficients. The quantity to be measured for individual monitoring is the personal dose equivalent, $H_{\mathrm{p}}(10)$ 
or $H_{\mathrm{p}}(0.07)$, respectively, in the body of the person wearing the dosemeter. For the calibration of personal dosemeters worn on the body, the true value of the quantity is given by the dose equivalent in an ICRU-tissue slab phantom at the depth specified by the quantity.

In order to determine the value of $H_{\mathrm{p}}(d)$ at the point of test, it is necessary to first determine the appropriate basic physical quantity

- air kerma for photon radiation,

- fluence for neutrons or

- absorbed dose for electrons

and then the corresponding conversion coefficient is used to calculate personal dose equivalent. Calibrations of personal dosemeters as well as measurements of their response as a function of energy and direction of radiation incidence should be carried out on the ISO water slab phantom ${ }^{(15-18)}$, a water-filled slab $\left(30 \times 30 \times 15 \mathrm{~cm}^{3}\right)$ and walls made of PMMA. The front wall should be $2.5 \mathrm{~mm}$ in thick and the other walls are of $10 \mathrm{~mm}$ thick. When this phantom is used, no corrections are applied for possible differences in backscatter between this phantom and the ICRU tissue slab phantom used to define the true value of the quantity. The personal dosemeter is fixed to the front face of the phantom so that the reference direction of the dosemeter coincides with the normal to the front face of the phantom. The reference point of the dosemeter is placed at the point of test.

For use in complex workplace radiation fields such as at high-energy accelerators ${ }^{(19,20)}$, which are significantly different from calibration fields (Figure 3), a normalisation factor may be needed to compensate for deficiencies of detectors in terms of angle and energy dependence of response ${ }^{(21-23)}$. Such normalisation factors can be obtained by field calibration in realistic radiation fields ${ }^{(24-26)}$. A welldocumented high-energy radiation field at $\mathrm{CERN}^{(27)}$ is often used to simulate realistic radiation fields around high-energy accelerators.

\section{INDIVIDUAL MONITORING TECHNIQUES}

\section{External photon/beta radiation}

There are three main techniques used for external dosimetry of photon/beta radiation:

- Photographic film

- Thermoluminescence (TLD)

- Optically stimulated luminescence (OSL).

Until some 30 years ago, film was the almost only technique used. Since then there has been a continuous tendency towards TLD. Recently, a significant shift from film to OSL has taken place in several countries.
On a smaller scale radio-photo-luminescence (RPL) dosemeters (mainly in Germany and Japan) and Direct Ion Storage dosemeters (DIS), e.g. at CERN, are in use. For extremity dosimetry, TLD is used in almost all cases.

Modern systems generally fulfil the technical requirements for individual monitoring for photon and beta radiation, with the exception of low-energy beta radiation, where only few systems are fully satisfactory $^{(28)}$.

\section{Neutron radiation}

For neutron dosimetry, two main techniques are used:

- Differential TLD measurements for thermal neutrons, e.g. ${ }^{6} \mathrm{LiF} /{ }^{7} \mathrm{LiF}$ (albedo) dosemeters.

- Track etch techniques, e.g. CR-39 for fast and high energy neutrons, and with converter also for thermal neutrons.

Until some 20 years ago NTA film was a widely used neutron detector, but production was not further guaranteed, urging some services to replace their systems. On smaller scales bubble detectors are used.

Individual monitoring for neutron radiation is still today a challenge. Energy dependence of the response, sensitivity and dynamic range are limiting factors of most available systems ${ }^{(28)}$. These deficiencies may be somewhat eased by the fact that neutron dose values are usually rather low.

\section{Examples of monitoring methods and techniques}

A radiation protection monitoring program includes many more purposes than just assessment of dose. Therefore, the methods have always been optimised to offer additional information besides dose. A strong argument for using film or OSL dosemeters was their capability to render information on the irradiation conditions (estimation of photon energy, angle of irradiation, movement during irradiation, etc.). Recent developments with electronic dosemeters add new options ${ }^{(29-32)}$. Most striking, the dose values can be made available immediately after the irradiation. With modern IT systems, this information is immediately accessible for all persons involved, allowing actions to be taken for specific actual situations in due time and the recorded dose can be attributed to work done in given time and given locations (so-called 'job-dosimetry').

At the Los Alamos National Laboratory (LANL), a system based on TLD for photon radiation and track etch for neutron radiation is in use $^{(33,34)}$. The characteristics of additional systems available at LANL, including TLD albedo detectors $\left({ }^{6} \mathrm{LiF} /{ }^{7} \mathrm{LiF}\right.$ pairs), see Figure 4 , bubble detectors 


\section{WERNLI}

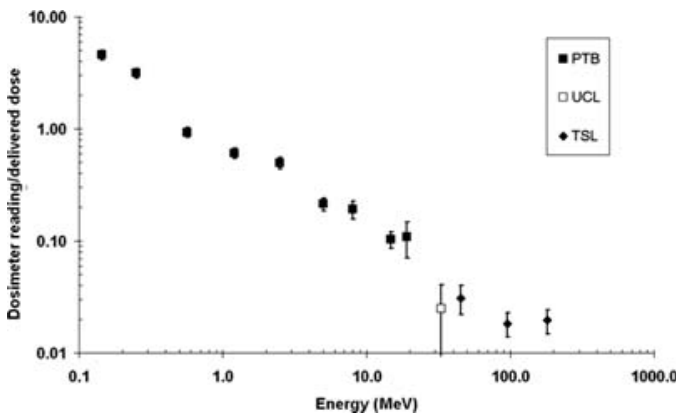

Figure 4. Energy dependence of the response of the albedo $\left({ }^{6} \mathrm{LiF} /{ }^{7} \mathrm{LiF}\right)$ neutron detectors tested at $\mathrm{LANL}^{(35)}$.

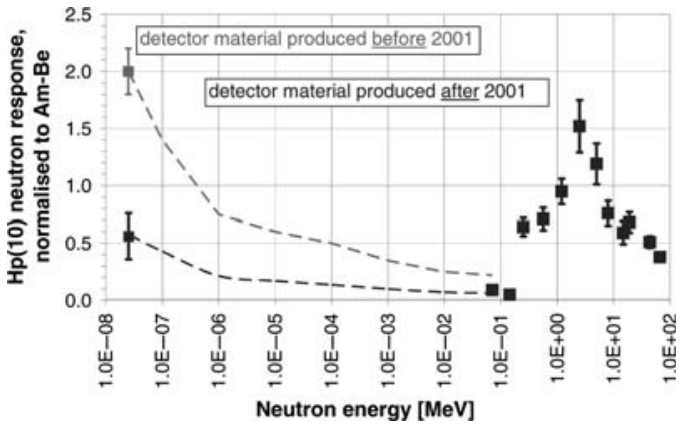

Figure 5. Energy dependence of the response of the CR-39 neutron detectors used at PSI and CERN.

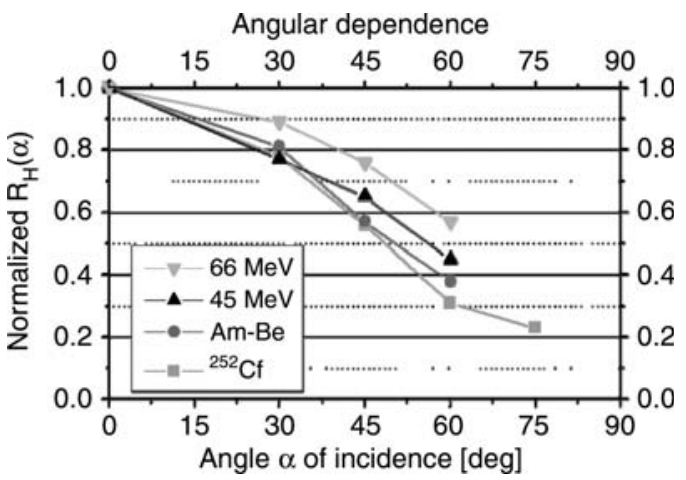

Figure 6. Angular dependence of the response of the CR39 neutron detectors used at PSI and CERN.

and different track etch detectors have been studied $^{(35)}$. For the track etch neutron detectors, results consistent with data published elsewhere, e.g. as shown in Figures 5 and 6, were found.

A system consisting of Direct Ion Storage (DIS-1) photon/beta detectors $^{(36)}$ and CR-39 neutron detectors ${ }^{(37)}$ has been introduced for use at $\mathrm{CERN}^{(38,39)}$.
(1) Every worker who possibly enters a radiation zone is equipped with a combined passive personnel dosemeter for the measurement of $H_{p}(10)$ and $H_{p}(0.07)$ for photon and beta radiation, as well as of $H_{p}(10)$ for neutron radiation.

(2) A number of local readout stations (e.g. $\sim 40$ for CERN) for the photon/beta dosemeters are distributed over the site. These stations display the accumulated dose locally and via LAN all readouts are registered automatically in a central database of the dosimetry service.

(3) The workers are asked to read their dosemeters periodically, e.g. at the beginning of each month at any station nearby. In addition, the workers are free to read their dosemeters as often as they like to do so.

(4) For job dosimetry a specific group of workers may be asked to read their dosemeters each time they enter or leave a given area.

(5) The dosemeters stay with the worker for up to $1 \mathrm{y}$. Every month about $10 \%$ of all dosemeters are exchanged. If the photon dose exceeds $2 \mathrm{mSv}$ per month, the dosemeter is immediately exchanged.

(6) On return of the dosemeter to the dosimetry service, the neutron detectors are removed and processed for readout. The photon/beta dosemeters are quality controlled, the dose cleared, the badge loaded with a new neutron detector and made available for new issuing.

(7) Neutron dose is booked in the year of readout of the neutron dosemeter if not exceptional conditions indicate otherwise.

(8) To compensate for the pronounced energy dependence of the response of the neutron detector (Figure 5), a normalisation factor of 1.5 is applied for use at CERN. The lowest dose reported is $0.5 \mathrm{mSv}$.

\section{OUTLOOK}

\section{Dosimetry for photon radiation}

This is the bulk of dosimetric activities worldwide, attracting commercial companies to take over and to control the market. Such large services have the advantage of well-developed and standardised methods and low cost for the customer. On the other hand, as a consequence of the commercial concentration of services, the local expertise in radiation dosimetry may diminish and flexibility for sitespecific solutions may become limited. It may also be not easy for national authorities to verify the quality of foreign services operating in their region of responsibility and to receive feedback requested according to national legislation.

The use of electronic devices as legal dosemeters in combination with the corresponding IT networks 
and software offers new options for individual monitoring. But only a small number of suitable systems are available on the market.

\section{Dosimetry for beta radiation}

The main application is extremity dosimetry. New designs of detectors comfortable to wear and less energy dependent are still needed. Active devices are still missing, but would attract some interest.

\section{Dosimetry for neutron radiation}

All available passive systems have some limitations and no immediate relieve is expected. New concepts $^{(40)}$, further improvements of detector materials, together with sophisticated converter materials, and evaluation procedures may remedy the existing constraints. Electronic neutron dosemeters are emerging on the market ${ }^{(32,41)}$. Their use may complement passive systems in various applications, but presumably not replace them.

Remaining challenges for individual monitoring at high-energy accelerators include improvements on energy and angular dependence of response, as well as sensitivity to (high energy) neutrons. For operational aspects, a quick dosemeter read out and, of course, low cost of the whole system would be desirable. Therefore, work remains to be done by a young generation in radiation dosimetry.

\section{REFERENCES}

1. ICRP 103. Recommendations of the International Commission on Radiological Protection. ICRP Publication 103. Ann. ICRP 37(2-4) (2007).

2. NCRP Report No. 144. Radiation Protection for Particle Accelerator Facilities (2003).

3. McDonald, J. C. Challenges of Personnel Dosimetry at Accelerators, in Topics in Accelerator Health Physics, Professional Development School, 31 January-2 February 2008 Oakland, CA. Medical Physics Publishing, Madison, WI.

4. ICRU 33. Radiation Quantities and Units. ICRU Report 33 (ICRU: Washington) (1980).

5. ICRU 39. Determination of Dose Equivalents Resulting from External Radiation Sources. ICRU Report 39 (Bethesda: ICRU Publications) (1985).

6. ICRU 43. Measurement of Dose Equivalents from External Radiation Sources, Part 2. ICRU Report 43 (Bethesda: ICRU Publications) (1988).

7. ICRU 51. Quantities and Units in Radiation Protection Dosimetry. ICRU Report 51 (Bethesda: ICRU Publications) (1993).

8. ICRP 60. Recommendations of the International Commission on Radiological Protection. ICRP Publication 60. Ann. ICRP 21(1-3) (1991).

9. ICRP 74. Conversion Coefficients for Use in Radiological Protection against External Radiation. ICRP Publication 74. Ann. ICRP 26(3-4) (1996).
10. Schlattl, H. et al. Organ dose conversion coefficients for voxel models of the reference male and female from idealized photon exposures. Phys. Med. Biol. 52, 2123-2145 (2007).

11. Pelliccioni, M. Radiation weighting factors and high energy radiation. Radiat. Prot. Dosim. 80(4), 371-378 (1998).

12. Pelliccioni, M. Overview of fluence-to-dose and fluenceto-ambient dose equivalent coefficients for high-energy radiation calculated using the FLUKA code. Radiat. Prot. Dosim. 88(4), 279-298 (2000).

13. Pelliccioni, M. The impact of ICRP publication 92 on the conversion coefficients in use for cosmic ray dosimetry. Radiat. Prot. Dosim. 109(4), 303-309 (2004).

14. IAEA Safety Reports Series No 16. Calibration of Radiation Protection Monitoring Instruments (2000).

15. ISO 4037. $X$ and gamma reference radiations for calibrating dosemeters and dose rate meters and for determining their response as a function of photon energy. International Organization for Standardization, Geneva, Switzerland.

16. ISO 4037-1. $\mathrm{X}$ and gamma reference radiations for calibrating dosemeters and dose rate meters and for determining their response as a function of photon energy. Part 1: Radiation characteristics and production methods. International Organization for Standardization, Geneva, Switzerland (1996).

17. ISO 4037-2. $X$ and gamma reference radiations for calibrating dosemeters and dose rate meters and for determining their response as a function of photon energy. Part 2: Dosimetry for radiation protection over the energy range $8 \mathrm{keV}$ to $1.3 \mathrm{MeV}$ and $4 \mathrm{MeV}$ to $9 \mathrm{MeV}$. International Organization for Standardization, Geneva, Switzerland (1997).

18. ISO 4037-3. X and gamma reference radiations for calibrating dosemeters and dose rate meters and for determining their response as a function of photon energy. Part 3: Calibration of area and personal dosemeters and the measurement of their response as a function of energy and angle of incidence, International Organization for Standardization, Geneva, Switzerland (1999).

19. Bilski, P. et al. Complex workplace radiation fields at European high-energy accelerator and thermonuclear fusion facilities, CERN-2006-007, Geneva (2006).

20. Buchillier, T. et al. Calibration and testing of a TLD dosemeter for area monitoring. Radiat. Prot. Dosim. 110(1-4), 705-710 (2004).

21. Spurny, F. Individual dosimetry for high energy radiation fields. Radiat. Prot. Dosim. 85(1-4), 15-20 (1999).

22. ICRU 66. Determination of operational dose equivalent quantities for neutrons. ICRU Report 66. J. ICRU 1(3) (2001).

23. Fiechtner, A., Boschung, M. and Wernli, C. Progress report of the CR-39 neutron personal monitoring service at PSI. Radiat. Prot. Dosim. 125(1-4) (2007).

24. Boschung, M., Fiechtner, A., Mayer, S. and Wernli, C. Field calibration and comparison of personal neutron dosemeter designs based on CR-39 for the use around high-energy accelerators. Radiat. Meas. 43(2-6) (2008).

25. Grecescu, M. et al. Neutron field measurements at the $590 \mathrm{MeV}$ ring cyclotron of the Paul Scherrer Institute. Nucl. Instrum. Meth. Phys. Res. A 476, 58-62 (2002).

26. Nolte, R. et al. High-energy neutron reference fields for calibration of detectors used in neutron spectrometry. Nucl. Instrum. Meth. Phys. Res. A 476, 369-373 (2002). 


\section{WERNLI}

27. Mitaroff, A. and Silari, M. The CERN-EU high-energy reference field (CERF) facility for dosimetry at commercial flight altitudes and in space. Radiat. Prot. Dosim. 102, 7-22 (2002).

28. European Radiation Dosimetry Group (EURADOS). Harmonisation and dosimetric quality assurance in individual monitoring for external radiation. Radiat. Prot. Dosim. 89(1-2) (2000).

29. Wernli, C. and Butterweck, G. Recent developments on electronic dosimeters. In: V Regional Congress on Radiation Protection and Safety, Recife, Brazil (2001).

30. Bolz, M. and Pécossais, P. La première solution complète pour une dosimétrie personnelle active: une technique d'avant-garde conçue pour l'utilisateur. Radioprotection 37(2) (2002).

31. Wahl, W. Wie wird, wie soll die Personendosimetrie der Zukunft aussehen? Wünsche und Forderungen aus der Praxis. StrahlenschutzPRAXIS, Heft 2/2002.

32. Luszik-Bhadra, M. Electronic personal neutron dosemeters for high energies: measurements, new developments and further needs. Radiat. Prot. Dosim. 126(14), 487-490 (2007).

33. Stewart, R. D., McDonald, J. C., Otto, T. and Loesch, R. M. Forth intercomparison of personal dosemeters used in US Department of Energy Accelerator Facilities. Radiat. Prot. Dosim. 87(2), 77-86 (2000).
34. Hoffman, J. M. and Mallet, M. W. The LANL model 8823 whole-body TLD and associated dose algorithm. Radiat. Protect. J. 77(5 Suppl.) (1999).

35. Olsher, R. et al. High-energy response of passive dosemeters in use at LANL. Radiat. Prot. Dosim. 126(14), 326-332 (2007).

36. Boschung, M., Fiechtner, A. and Wernli, C. A legally approved personal dosemeter for photon and beta radiation based on direct ion storage. Radiat. Prot. Dosim. 101(1-4), 271-274 (2002).

37. Fiechtner, A. and Wernli, C. Individual neutron monitoring with CR-39 detectors at an accelerator centre. Radiat. Prot. Dosim. 85(1-4), 35-38 (1999).

38. Wernli, C. and Boschung, M. A. Novel Concept and Technique for Individual Monitoring for Photon, Beta and Neutron Radiation. IRPA11 (2004).

39. Carbonez, P., Kotamäki, E. and Otto, T. The personal dosimetry service at CERN. Technical Note CERNSC-2008-056-RP-TN (2008).

40. Akselrod, G.M. et al. A novel Al2O3 fluorescent nuclear track detector for heavy charged particles and neutrons. Nucl. Instrum. Meth. Phys. Res. B 247, 295-306 (2006).

41. Schuhmacher, H. et al. Evaluation of individual dosimetry in mixed neutron and photon radiation fields. PTB-N-49 (2006). 